\title{
LXIII. The effective capacity of a pancake coil
}

\section{G. Breit}

To cite this article: G. Breit (1922) LXIII. The effective capacity of a pancake coil , Philosophical Magazine Series 6, 44:262, 729-740, DOI: 10.1080/14786441108634038

To link to this article: http://dx.doi.org/10.1080/14786441108634038

册 Published online: 08 Apr 2009.

Submit your article to this journal 정

Џll Article views: 5

Q View related articles ¿

4 Citing articles: 1 View citing articles ๘ 
LXIII. The Effective Capacity of a Pancake Coil. $B y$ G. Breit *.

Purpose.

T' has been shown in a previous paper t that the effective 1 capacity of a coil may be computed as

where

$$
\mathrm{C}_{0}=\int_{x_{\mathrm{I}}}^{x_{2}} \frac{\mathrm{M}(x)}{\mathrm{L}}\left\{\int_{x_{1}}^{x} \frac{\alpha(x)}{\mathrm{L}} d x\right\} d x, \ldots .
$$

$x$ is an arbitrary parameter along the wire ;

$\mathrm{L}$ is the inductance of the coil ;

$\mathrm{M}(x) d x$ is the mutual inductance of the section between $x$ and $x+d x$ to the rest of the coil ;

$\left(\frac{d i}{d t}\right) \alpha(x) d x$ is the charge on the element $d x, i$ being the current through the coil terminal;

$x_{1}, x_{2}$ are the values of $x$ at the coil terminals, the value $x_{1}$ corresponding to the ungrounded terminal of the coil.

The conditions which were assumed in deriving this formula are :-

(1) The constant $\mathrm{C}_{0}$ exists.

(2) The product of the frequency used into the conductivity is so high that the wire of the coil may be considered as a perfect conductor : i.e., the electric intensity is practically perpendicular to the surface of the wire at any instant.

(3) The dimensions of the coil are sufficiently small to make it legitimate to neglect the phase differences introduced into the retarded potentials by currents and charges in different portions of the coil.

(4) The formula still applies if $\mathrm{C}_{0}$ is not a constant in general, but is constant within a range beginning at very low frequencies.

It is the purpose of this paper to apply this formula to the case of a pancake coil.

* Conmunicated by the Director of the Bureau of Standards, Washington.

† See "The Distributed Capacity of Inductance Coils," by G. Breit, Phys. Rev. xviii. p. 649 (1921). 
By a pancake coil is meant a coil whose wires are all wound in one plane in a spiral, as shown on the figure (see fig. 1). It will be supposed that the number of turns Fig. 1.--Pancake coil.

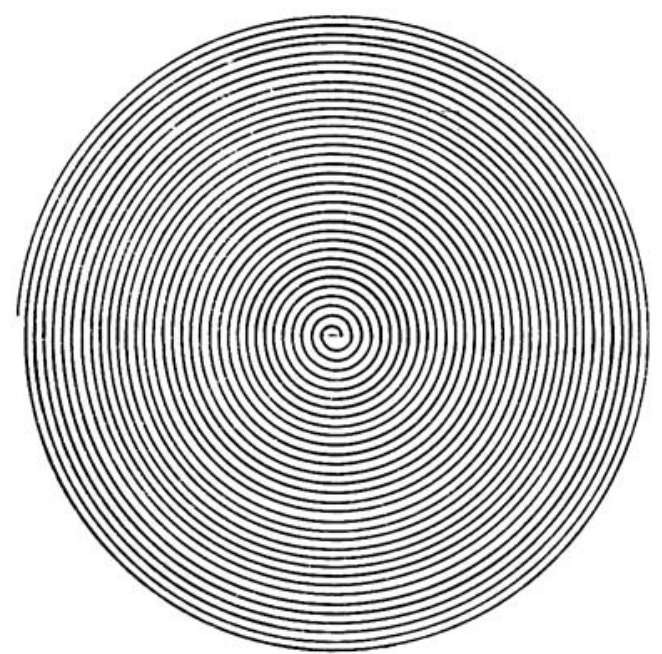

in the coil is large, that the turns are close together, and that the thickness of the insulation is negligible.

Thus the coil may be replaced by a disk on whose surface the potential varies in the same manner as it does in the coil.

\section{Notation.}

The radius of the pancake will be denoted by $a$.

Points in space will be referred to by cylindrical coordinates

$$
(r, z, \theta) \text {, }
$$

with centre $O$ at the centre of the coil, and with axis perpendicular to the plane of the coil.

Fig. 2.--Cross section of pancake coil by plane through diameter.

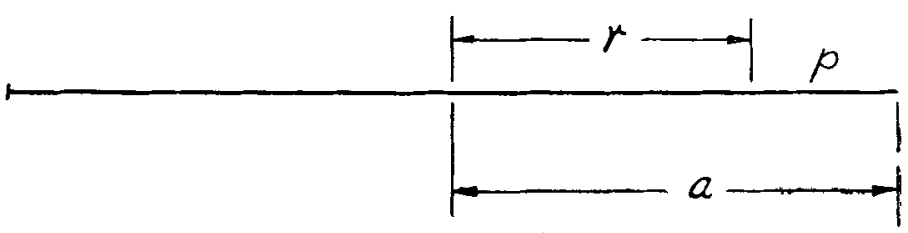

The meaning of these symbols is shown on fig. 2 . 
Simplifying Assumption as to Potential Listribution.

An arithmetical computation of the e.m.f. induced in various parts of the coil for a coil with a finite number of turns revealed the fact that the e.m.f. induced between a point on the surface of the coil and the centre is approximately proportional to the square of the distance of that point from the centre. The computation above mentioned consisted in calculating the e.m.f. induced between the centre and a number of points at various distances from the centre for the case of a coil having a finite number of equally spaced turns. Maxwell's formula in elliptic integrals was used, and numerical results were tabulated. These were then plotted, and the graph revealed the approximate relation stated.

The relation is frankly approximate, but is believed to be accurate enough for the calculation of the coil capacity. The computation which follows takes this for its startingpoint.

\section{General Plan of Attack.}

The first step will be to compute the distribution of charge on the wires of the coil which will satisfy the law assumed for the potential distribution. Then the quantity $\mathbf{M}(x)$ will be determined from the same law. The two expressions will next be substituted in (1), and hence $\mathrm{C}_{0}$ will be obtained.

This will be done for three cases-namely that of the coil when ungrounded, and also when grounded-either at the centre or else at the outer edge.

The first part of the work consists, then, in the solution of an electrostatic problem-namely that of finding the charge distribution. The second part is ordinary integration.

\section{Solution of the Electrostatic Problem.}

It is convenient to transform the cylindrical co-ordinates to elliptical co-ordinates

$$
(r, z, \theta)
$$

by the formula

$$
(u, v, \theta)
$$

$$
\text { where } \left.\begin{array}{rl}
r+j z & =a \cosh (u+j v), \\
\text { or its equivalents } \quad . \quad . \quad & =\sqrt{-1}, \\
r & =a \cosh u \cos v, \\
z & =a \sinh u \sin v .
\end{array}\right\} . . \quad \text {. }
$$


The surface $u=$ constant gives a spheroid of revolution whose equation is

$$
\frac{r^{2}}{a^{2} \cosh ^{2} u}+\frac{z^{2}}{a^{2} \sinh ^{2} u}=1, \quad . \quad \text {. . . }
$$

as is seen by eliminating $v$ from (3); and the surface $v=$ constant gives a hyperboloid of revolution whose equation is

$$
\frac{r^{2}}{a^{2} \cos ^{2} v}-\frac{z^{2}}{a^{2} \sin ^{2} v}=1, . \quad . \quad . \quad . \quad .
$$

as is seen by eliminating $u$ from $(3)$.

The two sets of surfaces represented by (4) and (5) are orthogonal because (2) is a conformal transformation. Also the planes $\theta=$ constant are perpendicular to both (4) and $(5)$. Thus the co-ordinates $(u, v, \theta)$ are orthogonal.

It is readily shown that the Laplacian in these coordinates is

$$
\begin{aligned}
\frac{1}{\cosh u} \frac{\partial}{\partial u}\left(\cosh u \frac{\partial V}{\partial u}\right) & +\frac{1}{\cos v} \frac{\partial}{\partial v}\left(\cos v \frac{\partial V}{\partial v}\right) \\
& +\frac{\cosh ^{2} u-\cos ^{2} v}{\cosh ^{2} u \cos ^{2} v} \cdot \frac{\partial^{2} V}{\partial \theta^{2}}=0 .
\end{aligned}
$$

In particular, if $\mathrm{V}$ is independent * of $\theta$,

$$
\nabla^{2} V=\frac{1}{\cosh u} \frac{\partial}{\partial u}\left(\cosh u \frac{\partial V}{\partial u}\right)+\frac{1}{\cos v} \frac{\partial}{\partial v}\left(\cos v \frac{\partial V}{\partial v}\right)
$$

* This expression may be derived by remembering that if

$$
x_{1}, \quad x_{2}, \quad x_{3}
$$

are three orthogonal co-ordinates of such a kind that the differentials of length corresponding to the three differentials

are

$$
d x_{1}, \quad d x_{2}, \quad d x_{3}
$$

$$
\frac{d x_{1}}{h_{1}}, \frac{d x_{2}}{h_{2}}, \frac{d x_{3}}{h_{3}}
$$

then

$$
\begin{aligned}
\nabla^{2} V=h_{1} h_{2} h_{3}\left\{h_{1} \frac{\partial}{\partial x_{1}}\left(\frac{h_{1}}{h_{2} h_{3}} \frac{\partial V}{\partial x_{1}}\right)\right. & +h_{2} \frac{\partial}{\partial x_{2}}\left(\frac{h_{2}}{h_{1} h_{3}} \frac{\partial V}{\partial x_{2}}\right) \\
& \left.+h_{3} \frac{\partial}{\partial x_{3}}\left(\frac{h_{3}}{h_{1} h_{2}} \frac{\partial V}{\partial x_{3}}\right)\right\} .
\end{aligned}
$$

See W. E. Byerly, 'Fourier Series and Spherical Harmonics,' p. 239, equation (6). 
It is advantageous to transform this by

$$
\left.\begin{array}{l}
\mu=\sin v, \\
\nu=j \sinh u,
\end{array}\right\} . .
$$

which reduces (6) to

$$
\nabla^{2} V=-\frac{\partial}{\partial \nu}\left\{\left(1-\nu^{2}\right) \frac{\partial V}{\partial \nu}\right\}+\frac{\partial}{\partial \mu}\left\{\left(1-\mu^{2}\right) \frac{\partial V}{\partial \mu}\right\} .
$$

Now the electrostatic problem to be solved is that of finding for $\mathrm{V}$ a solution which together with its first derivatives is finite and continuous, which is independent of $\theta$, which satisfies the Laplacian

$$
\nabla^{2} \mathrm{~V}=0 \text {. }
$$

which vanishes at infinity at least to the first order and which at the disk becomes

$$
\mathrm{V}=\mathrm{V}_{0}-\mathrm{L} \frac{r^{2}}{a^{2}} \frac{d i}{d t} . \quad . \quad . \quad . \quad .
$$

But the equation of the disk is

$$
u=0 \text {; }
$$

in which case (3) reduces to

$$
r=a \cos v \text {. }
$$

Hence, using (7), equation (10) becomes

$$
\mathrm{V}=\mathrm{V}_{0}-\mathrm{L}\left(1-\mu^{2}\right) \frac{d i}{d i} \cdot \quad . \quad . \quad .
$$

Now the expression

$$
\left[\alpha_{n} \mathrm{P}_{n}(\nu)+\beta_{n} \mathrm{Q}_{n}(\nu)\right]\left[\alpha_{n} \mathrm{P}_{n}(\mu)+b_{n} \mathrm{Q}_{n}(\mu)\right]
$$

where $\mathrm{P}_{n}, \mathrm{Q}_{n}$ are Legendre functions of the first and second kind respectively, when substituted in (9) satisfies (9) in virtue of $(8)$. If, then, one should be able to find such values of $\alpha_{n}, \beta_{n}, a_{n}, b_{n}$, and such values of $n$ that

$$
\mathrm{V}=\sum_{n}\left[\alpha_{n} \mathrm{P}_{n}(v)+\beta_{n} \mathrm{Q}_{n}(\nu)\right]\left[a_{n} \mathrm{P}_{n}(\mu)+b_{n} \mathrm{Q}_{n}(\mu)\right]
$$

should vanish at infinity to the first order and should degenerate into (11) when $\nu$ approaches zero along the axis of pure imaginaries, then, in virtue of the uniqueness of the solution of (9) for given boundary conditions, the summation written gives the value of $\mathrm{V}$.

If the summation written is an infinite series it also gives V, provided it is universally convergent as to $\mu$ and $v$. 
Further, for a given $v, V$ may be represented by a series of the form

$$
\sum_{n=0}^{\infty} \mathrm{A}_{n} \mathrm{P}_{n}(\mu)
$$

the summation being taken over all positive integral values of $n$, because $V$ obviously satisfies the conditions which make such an expansion legitimate. The coefficient $A_{n}$ is independent of $\mu$ but, for different values of $\nu$, varies and is thus a function of $\nu$. It must be clearly of the form

$$
\mathrm{A}_{n}=\alpha_{n} \mathrm{P}_{n}(\nu)+\beta_{n} \mathrm{Q}_{n}(\nu),
$$

for otherwise (9) would not be satisfied. Here $n$ is a positive integer. The function $\mathrm{P}_{n}(v)$ is therefore a finite polynominal *, viz.

$$
\begin{aligned}
\mathrm{P}_{n}(\nu)=\frac{1.3 .5 \ldots(2 n-1)}{1.2 .3 \ldots \ldots} & {\left[\nu^{n}-\frac{n(n-1)}{2(2 n-1)} \nu^{n-2}\right.} \\
& \left.+\frac{n(n-1)(n-2)(n-3)}{2 \cdot 4(2 n-1)(2 n-3)} \nu^{n-4}-\ldots\right],
\end{aligned}
$$

and $Q_{n}(\nu)$ is an infinite series when $|\nu|>1$, viz.

$$
\begin{aligned}
Q_{n}(\nu)= & \frac{1.2 \ldots n}{1.3 .5 \ldots(2 n+1)}\left\{\frac{1}{v^{n+1}}+\frac{(n+1)(n+2)}{2(2 n+3)} \frac{1}{v^{n+3}}\right. \\
& \left.+\frac{(n+1)(n+2)(n+3)(n+4)}{2.4(2 n+3)(2 n+5)} \frac{1}{v^{n+5}}+\ldots . \ldots\right\} .
\end{aligned}
$$

But points at infinite distance from the origin are given by real, positive, infinitely large values of $u$, and consequently in accordance with (7) by infinitely large values of $v$ on the positive half of the axis of pure imaginaries. Such values can be denoted as usual by $+j \infty$. It is clear that if $n>0$, the expression for $P_{n}(\nu)$ becomes infinite for $\nu=+j \infty$ because it is a sum of terms of the same sign, and each term becomes infinite. Hence, if $n<0, \alpha_{n}=0$.

Further, there is symmetry about the plane $z=0$. Hence by (3) and (7) only even values of $n$ can be taken. Thus the most general possible expression for $\mathrm{V}$ is

$$
\mathrm{V}=\sum_{n=1}^{\infty} a_{2 n} \mathrm{P}_{2 n}(\mu) \mathrm{Q}_{2 n}(\nu) . \quad . \quad \cdot
$$

The coefficionts $A, a_{2 n}$ must now be determined in such a way that

$$
\sum_{n=0}^{\infty} a_{2 n} \mathrm{P}_{2 n}(\mu) \mathrm{Q}_{2 n}(j \cdot 0) \equiv \mathrm{V}_{0}-\mathrm{L}\left(1-\mu^{2}\right) \frac{d i}{d t}, \ldots
$$

* See W. F. Byerly, 'Fourier Series and Spherical Harmonics,' p. 145, equations (9) and (10). 
where the symbol $Q_{2 n}(j .0)$ stands for the limit of $Q_{2 n}(j . \delta)$ as $\delta$ approaches zero taking only real and positive values.

Since now

$$
1-\mu^{2}=\frac{2}{3}\left[\mathrm{P}_{0}(\mu)-\mathrm{P}_{2}(\mu)\right],
$$

all $a_{2 n}$ but $a_{0}, a_{2}$ vanish, and $a_{0}, a_{2}$ are determined by the relations

Thus

$$
\left.\begin{array}{l}
a_{0} \mathrm{Q}_{0}(j \cdot 0)=\mathrm{V}_{0}-\frac{2}{3} \mathrm{~L} \frac{d i}{d t}, \\
a_{2} \mathrm{Q}_{2}(j .0)=\operatorname{2}_{3} \mathrm{~L} \frac{d i}{d t}
\end{array}\right\} \cdot . \cdot .
$$

$$
\mathrm{V}=\frac{\mathrm{V}_{0}-\frac{2}{3} \mathrm{~L} \frac{d i}{d t}}{\mathrm{Q}_{0}(j \cdot 0)} \mathrm{Q}_{0}(j \cdot \sinh u)+\frac{2}{3} \mathrm{~L} \frac{d i}{d t} \frac{\mathrm{Q}_{2}(j \cdot \sinh u)}{\mathrm{Q}_{2}(j \cdot 0)} \mathrm{P}_{2}(\mu)
$$

The surface density of charge in coulombs is obtained as

$$
\sigma=-\frac{10^{-11}}{8} \cdot \overline{\mathrm{g}} \mathrm{K} \frac{\mathrm{K}}{4 \pi} \frac{\partial \mathrm{V}}{\partial n}
$$

where $\mathrm{K}$ is the dielectric constant of the medium, and $\frac{\partial \mathrm{V}}{\partial n}$ is the directional derivative of $V$ with respect to the normal drawn away from the surface. The same may be written as

$$
\left.\begin{array}{c}
\sigma=-\frac{\kappa}{4 \pi} \frac{\partial V}{\partial n}, \\
\text { where } \quad \kappa=\frac{10^{-11}}{8 \cdot 989} \mathrm{~K} .
\end{array}\right\} . . .
$$

Now at the disk the normal is parallel to OZ. Hence

$$
\frac{\partial V}{\partial n}=\left(\frac{\partial V}{\partial z}\right)_{z=0} \text { if } z>0 \text { and }-\left(\frac{\partial V}{\partial z}\right)_{z=0} \text { if } z<0 \text {. }
$$

Hence by (3)

$$
\frac{\partial V}{\partial n}=\frac{1}{a \mid \sin v_{1}}\left(\frac{\partial V}{\partial u}\right)_{u=0}
$$

and by (15), (16)

$$
\begin{aligned}
\sigma=-\frac{\kappa}{4 \pi a|\mu|}\left\{\left(\mathrm{V}_{0}\right.\right. & \left.-\frac{2}{3} \mathrm{~L} \frac{d i}{d t}\right) \frac{j \mathrm{Q}_{0}(j .0)}{\mathrm{Q}_{0}(j \cdot 0)} \\
& \left.+\frac{2}{3} \mathrm{~L} \frac{d i}{d t} \frac{j \mathrm{Q}_{2}^{\prime}(j .0)}{\mathrm{Q}_{2}(j \cdot 0)} \mathrm{P}_{2}(\mu)\right\} .
\end{aligned}
$$


But it may easily be shown that *

and

$$
\frac{j \mathrm{Q}_{0}{ }^{\prime}(j .0)}{\mathrm{Q}_{0}(j \cdot 0)}=-\frac{2}{\pi}
$$

Hence

$$
\frac{j Q_{2}{ }^{\prime}(j \cdot 0)}{Q_{2}(j \cdot 0)}=-\frac{8}{\pi} \text {. }
$$

$$
\sigma=\frac{\kappa}{2 \pi^{2} a|\mu|}\left\{\left(\mathrm{V}_{0}-\frac{2}{3} \mathrm{~L} \frac{d i}{d t}\right)+{ }_{3}^{8} \mathrm{I} \frac{d i}{d t} \mathrm{P}_{2}(\mu)\right\} .
$$

This solves the electrostatic problem proposed.

\section{Computation of the Function $\mathrm{M}(x)$.}

In order to find the function $M(x)$, a choice must be made of the variable $x$. Here $r$ will be chosen as this variable.

It was assumed that the e.m.f. varies as $r^{2}$. This means that

or, differentiating,

$$
\int_{0}^{r} \mathrm{M}(r) d r=\mathrm{L} \frac{r^{2}}{a^{2}}
$$

$$
M(r)=2 \mathrm{~L} \frac{r}{a^{2}} \cdot \cdot \cdot \cdot \cdot \cdot
$$

* These formulas can be derived from using the following facts :-

$$
\begin{aligned}
Q_{0}(z) & =\frac{1}{2} \log \frac{z+1}{z-1} . \\
\frac{1}{y-z} & =\Sigma(2 n+1) \mathrm{P}_{n}(z) Q_{n}(y) .
\end{aligned}
$$

(c) The recurrence formulas

$$
(2 n+1) z \mathrm{P}_{n}(z)=(n+1) \mathrm{P}_{n+1}(z)+n \mathrm{P}_{n-1}(z)
$$

Using (c) in (b), the expression for $\mathbf{Q}_{2 m}$ in terms of $\mathbf{Q}_{0}$ can be derived by writing the identity

$$
\frac{y}{y-z}-\frac{z}{y-z}=1 \text {. }
$$

Then from (a) expressions for $Q_{2 m}$ are obtained. On differentiating these expressions and passing the limit in the result of the differentiation as well as the original, the result follows at once. 
Computation of the Function $\alpha(x)$.

As stated in the in1roduction, the differential of charge is

$$
\frac{d i}{d t} \alpha(x) \cdot d x \text {. }
$$

The independent variable here chosen is $r$. As $r$ varies from 0 to $a$, and as $\theta$ varies from 0 to $2 \pi$, the whole coil is traversed by the point $(r, \theta)$. The differential of area is $2 \pi r d r$, and the differential of charge is then $4 \pi \sigma r d r$, where $\sigma$ is given by (17) becau e expression (17) gives the surface density only on one side of the coil.

By (3), on the coil $r$ becomes $a \cos v$; so that

$$
\mu=\sqrt{1-\cos ^{2} v}=\sqrt{1-\frac{r^{2}}{a^{2}}} \text {. }
$$

Substituting this in (3), and expressing the fact that

it is found that

$$
4 \pi \sigma r d r=\left(\frac{d i}{d t}\right) \alpha(r) d r
$$

$$
\alpha(r)=\frac{2 \kappa r \mathrm{~L}}{\pi \sqrt{a^{2}-r^{2}}}\left\{\left(\begin{array}{c}
V_{0} \\
\left(\frac{d i}{d t}\right) \mathrm{L}
\end{array}-\frac{2}{3}\right)+\frac{8}{3} \mathrm{P}_{2}\left(\sqrt{1-\frac{r^{2}}{a^{2}}}\right)\right\}
$$

It now remains to substitute (18), (19) into (1). If the ungrounded condenser terminal is connected to the centre, $x_{1}$ is to be taken as 0 and $x_{2}$ is to be taken as $a$. If, however, it is connected to the periphery, $x_{1}$ is to be taken as $a$ and $x_{2}$ as 0 .

The first of these gives

$$
\mathrm{C}_{0}=\frac{4 \kappa a}{\pi}\left[\frac{1}{15}+\frac{1}{6} \frac{\mathrm{V}_{0}}{\mathrm{~L} \frac{a i}{d t}}\right], \quad . \quad . \quad . \quad .
$$

and the second

$$
\mathrm{C}_{0}=\frac{4 \kappa a}{\pi}\left[\frac{2}{5}-\frac{1}{3} \frac{\mathrm{V}_{0}}{\mathrm{~L}} \frac{\mathrm{d} i}{d t}\right]
$$

If, now, the coil should be used with the centre grounded, and the ungrounded terminal of the condenser should be connected to the periphery, formula (21) applies, and in that formula $\nabla_{0}=0$. This gives

$$
\mathrm{C}_{0}=\frac{8 \kappa a}{5 \pi} \cdot \text {. . . . . . . }
$$

This is the effective capacity if the centre is grounded.

Phil. Mag. S. 6. Vol. 44. No. 262. Oct. 1922. 3 B 
Again, if the periphery is grounded and the centre is not, formula (20) applies, and in that formula $\mathrm{V}_{0}=\mathrm{L} \frac{d i}{d t}$, as is seen from (10) by setting $\mathrm{V}=0$ whən $r=a$. This gives

$$
\mathrm{C}_{0}=\frac{14 \kappa a}{15 \pi} \text {. . . . . . . }
$$

for the capacity with periphery grounded.

Finally, if the coil is insulated and the condenser is unshielded, as much current enters the coil as leaves it; so that (20) and (21) must give the same value for $\mathrm{C}_{0}$. that

Multiplying (20) by 2 and adding to (21), it is found

$$
\mathrm{C}_{0}=\frac{32 \kappa a}{45 \pi} \cdot \cdot \cdot \cdot \cdot \cdot \cdot
$$

if the coil is ungrounded.

It is worth mentioning that if $\mathrm{C}_{0}$ be eliminated from (20) and (21), it is found that $\frac{\mathrm{V}_{0}}{\mathrm{~L} \frac{d i}{d t}}=\frac{2}{3}$, which, in virtue of the identity

$$
\int_{0}^{1} \mathrm{P}_{2}(\mu) d \mu=0
$$

shows in a different way that the coil is insulated. is

Expressing the results in micromicrofarads, the capacity when grounded at centre....... $0.567 \mathrm{Ka} \mu \mu \mathrm{f}$, when grounded at periphery ... $0.330 \mathrm{~K} a \mu \mu \mathrm{f}$, when insulated ................ $0.252 \mathrm{~K} a \mu \mu \mathrm{f}$.

Now, according to the results of a previous calculation *, the effective capacity of a pancake coil of small depth when insulated is $0.437 \mathrm{Ka}$.

Thas, so far as the effective capacity i; concerned, there is an advantage in using pancake coils of large depth as compared to pancake coils of small depth.

\section{Experimental Verification.}

The formulas (22), (23) have been verified experimentally on a coil which is shown in fig. 3 . This coil is not circular

* See G. Breit, l. c. 
but hexagonal. The quantity $a$ is therefore not quite certain.

In the computations it was taken as the mean of the radii of the inscribed and escribed circles, which are $26.5 \mathrm{cms}$. and $29.5 \mathrm{cms}$. respectively. Hence the mean is $28 \mathrm{cms}$. The dielectric being air, the capacity with centre grounded should be $16 \mu \mu \mathrm{f}$, and if grounded at the periphery it should be $9 \mu \mu \mathrm{f}$. The values as measured are $16 \mu \mu \mathrm{f}$ and $9 \mu \mu \mathrm{f}$.

Fig. 3.---Photograph of pancake coil.

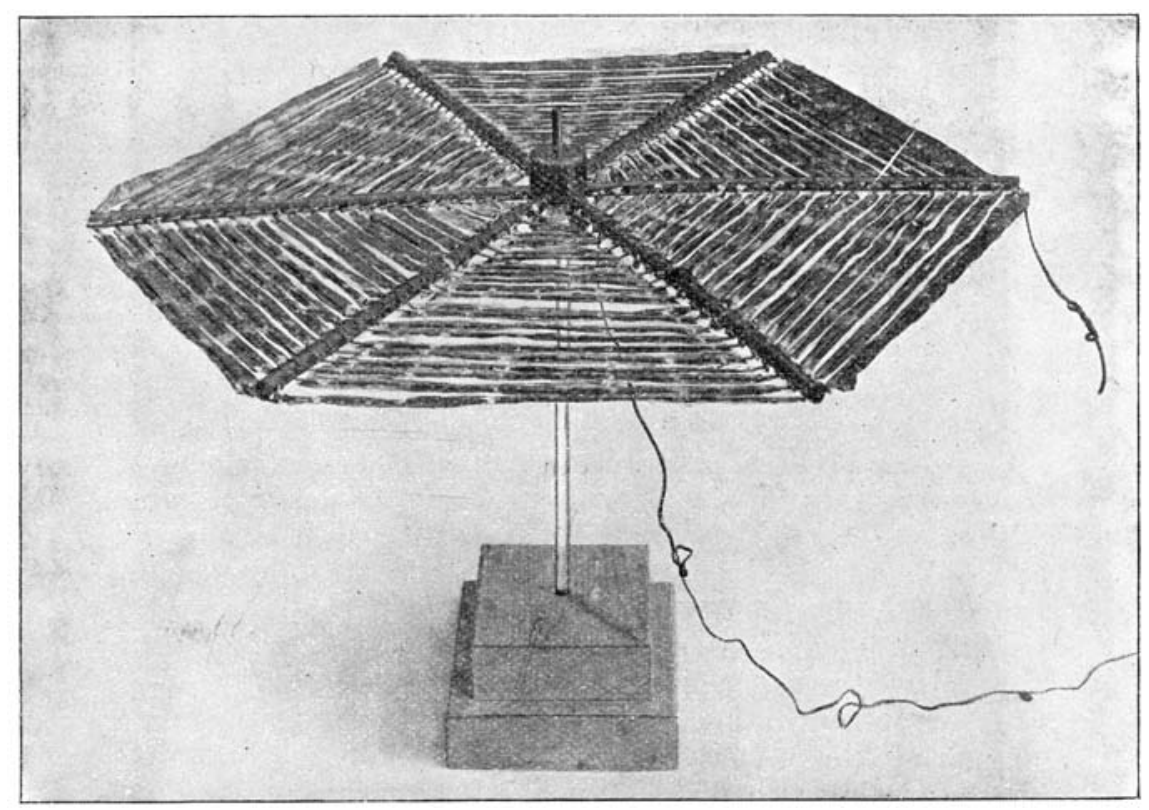

Measurements were not made more accurately than $1 \mu \mu \mathrm{f}$, on account of the difficulties connected with such measurements.

The capacity of the same coil was also measured without the copper foil, leaving only the copper braid. No change was detected in the capacity.

It also appeared that the copper rods used in fastening the braid could affect the capacity. A row of them was soldered to the braid, but no detectable change in capacity was noticed. 


\section{Conclusion.}

The effective capacity of a pancake coil has been calculated, and the calculations have been verified experimentally in two cases.

The results of the calculation are that the capacity of the coil

$$
\begin{aligned}
& \text { when grounded at centre is ....... } 0.567 \mathrm{Ka} \mu \mu \mathrm{f} \text {, } \\
& \text { when grounded at periphery is } \ldots 0.330 \mathrm{~K} \alpha \mu \mu \mathrm{f} \text {, } \\
& \text { when insulated is ................ } 0.252 \mathrm{~K} a \mu \mu \mathrm{f} \text {, }
\end{aligned}
$$

where $a$ is the radius of the coil and $K$ is the dielectric: constant of the medium.

W ashington, D.C..

$$
\text { Jan. 14, } 1922 .
$$

LXIV. The Relativity-Contraction in a Rotating Shaft moving. with Uniform Speed along its Axis. By FELIX $\mathrm{E}^{\circ}$ Hacketт, M.A., Ph.D., Professor of Physics, College. of Science for Ireland, Dublin *.

\section{\$1. Introduction and Summary.}

A SOLUTION is offered in this paper of the problem of A. the relativity-contraction in a rotating shaft moving with uniform velocity along its own axis. The standpoint adopted is that of the fixed æther and the FitzGerald-Lorentz contraction combined with the restricted principle of relativity. The validity of Euclidean geometry is assumed throughout the paper.

A hypothetical modification of Fizeau's method for measuring the velocity of light is considered-a rotating. shaft carrying two disks with apertures which correspond to the toothed wheel in Fizeau's experiment. It follows. readily that when a rotating shaft is moving with uniform velocity along its own axis, to a stationary observer, looking. in the direction of motion, it appears twisted in the opposite. sense to the rotation. This effect has been pointed out by R. W. Wood ${ }^{+}$, and he has discussed the experiment, but not in a sufficiently precise way to serve as a basis for the subsequent discussion in this paper.

The arrangement may act as a clock. It measures time

* Communicated by the Author. Read at the meeting of the British Association, September 1921.

† Woud,'Physical Optics,' 2nd edit. p. 690. 